\title{
CONSTRUCCIÓN DE INDICADORES DE CIENCIA, TECNOLOGÍA E INNOVACIÓN EN NICARAGUA
}

\section{CONSTRUCTION OF INDICATORS OF SCIENCE, TECHNOLOGY AND INNOVATION IN NICARAGUA}

\author{
Alemán Freddy S., Lezama Lilliam de Jesus \\ ${ }^{1}$ Universidad Nacional Agraria, Dirección de Investigacion, Extensión y Posgrado / freddy@una.edu.ni / llezama@una.edu.ni
}

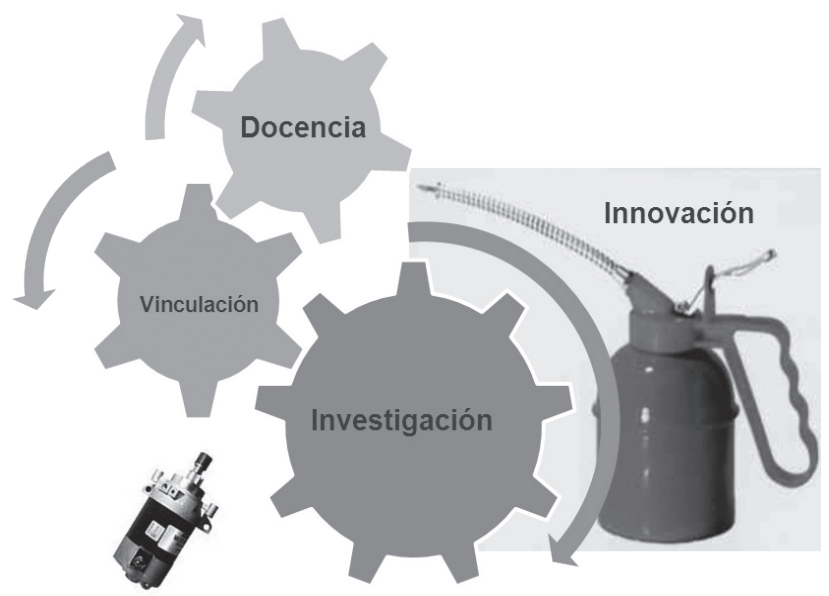

\section{RESUMEN}

Por primera vez en Nicaragua se están haciendo esfuerzos formales y diáfanos para irrumpir en la sociedad de la información y el conocimiento. En el año 2010, el Consejo Nicaraguenses de Ciencia y Tecnología (CONICYT) en colaboración con agentes claves de los sistemas de investigación e innovación definió el Plan Nacional de Ciencia, Tecnología e Innovación, Nicaragua, 2010-2013, el cual cuenta dentro de sus programas estratégicos el desarrollo de un sistema de indicadores de Ciencia, Tecnología e Innovación que oriente la definición de políticas y la toma de decisiones en materia científica. En la actualidad, el CONICYT en colaboración con el Consejo Nacional de Universidades (CNU) están implementado un estudio encauzado a la definición de un Sistema Nacional de Indicadores que permita disponer de información confiable y actualizada sobre Ciencia, Tecnología e Innovación en Nicaragua, que brinde información sobre inversión y gasto en Ciencia y Tecnología, la oferta científica - tecnológica nacional, el impacto del gasto público en C,T e I, y la forma como éste gasto se traduce en bienes y servicios para la sociedad nicaragüense. El proceso de construcción de dichos indicadores se ha estado realizando a través de la utilización de diversas técnicas y metodologías participativas, las cuales han estado siendo aplicadas por etapas, elaboración del marco conceptual; formulación de indicadores; elaboración de documento y aprobación del documento final. El sistema nacional de indicadores estará completo en la medida que los actores claves del sistema nacional de investigación e innovación se interesen en la creación del mismo, esto conlleva analizar la importancia de la creación del sistemas a la luz de su aplicabilidad práctica y el intercambio de experiencias con otros países de la región que han desarrollado el

\section{ABSTRACT}

For the first time in Nicaragua, a formal and diaphanous effort is being made to break into the information and knowledge society. In 2010, the National Council for Science and technology (CONICYT) in collaboration with key actors from the Nicaraguan research and innovation systems defined the National Plan for Science, Technology and Innovation, Nicaragua 2010-2013, which in one of its strategic programs concerns the development of a system of indicators for Science, Technology and Innovation, to guide policymaking and decision-making in science. CONICYT in collaboration with the National Council of Universities are implementing a study focused to the definition of a national system of indicators to provide reliable and updated information on Science, Technology and Innovation in Nicaragua. The systems will provide information on investment and spending on science and technology, the national scientific and technological offer, the impact of public expenditure on $\mathrm{S} \& \mathrm{~T}$ and how this expenditure has resulted in goods and services to Nicaraguan society. The building process of the indicators has been completed through the use of various techniques and participatory methodologies, which have been implemented in stages. The stages include the development of a conceptual framework, formulation of indicators, preparation of a report and approval of the final report. The national system of indicators will be completed to the extent that the key actors of the national research and innovation system are interested in creating the system of indicators, this involves the analysis of the importance of the creation of indicator systems in light of their practical applicability, and the exchange of experiences with other countries in the region that have developed the process. It is necessary to create compromises between the 
proceso. Es necesario el compromiso de los integrantes del sistema de investigación e innovación de proporcionar la información y alimentar el sistema, generar un software para administrar el sistema, y capacitar personal que estará a cargo de la recolección sistemática de la información, análisis, elaboración de reportes, comparaciones con otros países, cienciometría, así como percepción pública de la ciencia e impacto social del conocimiento.

Palabras clave: Investigacion científica innovación tecnológica bibliometría, impacto social de la ciencia.

Abreviaturas: CTI, Ciencia, Tecnología e Innovación; CONICYT, Consejo Nicaraguenses de Ciencia y Tecnología, CNU, Consejo Nacional de Universidades; SNII, Sistema Nacional de Investigacion e Innovación; I+D+i; Investigacion, Desarrollo e Innovación.

$\mathrm{P}$ or primera vez en Nicaragua se están haciendo esfuerzos formales y diáfanos para irrumpir en la sociedad de la información y el conocimiento. Los problemas que aquejan a nuestra sociedad, como la pobreza, la exclusión social, el insuficiente desarrollo industrial y el bajo valor agregado de nuestra producción, promueven la necesidad de ubicar Ciencia y Tecnología al servicio del desarrollo económico y social de Nicaragua.

Algunas acciones esperanzadoras en materia de Ciencia, Tecnología e Innovación en Nicaragua son la institucionalización de las acciones de Ciencia y Tecnología, a través de la creación del Consejo Nicaraguenses de Ciencia y Tecnología (CONICYT), la conformación de la Académica de Ciencias de Nicaragua, la definición de prioridades de investigación del Gobierno Central, el análisis de correspondencia entre líneas de investigación universitarias y líneas priorizadas en el Plan Nacional de Desarrollo Humano, y el fomento a la publicación científica nacional, entre otras.

En agosto del año 2010, CONICYT con la colaboración técnica de la Comisión Económica para América Latina y el Caribe (CEPAL) de las Naciones Unidas y un grupo de consultores nacionales e internacionales definió el Plan Nacional de Ciencia, Tecnología e Innovación, Nicaragua, 2010-2013 que tiene como propósito impulsar el desarrollo económico y social sostenible de Nicaragua, cuyos motores principales son la Ciencia, la Tecnología y la Innovación. En este plan, uno de los programas estratégicos se refiere al desarrollo de un sistema de indicadores de ciencia, tecnología e innovación para fortalecer el proceso de diseño y ejecución de políticas de Ciencia, Tecnología e Innovación y la toma de decisiones de los actores del Sistema Nacional de Investigadores de Nicaragua.

Es por ello que en la actualidad, el CONICYT, en colaboración con el Consejo Nacional de Universidades, están implementado un estudio dirigido a la definición de un sistema de indicadores que permita disponer de información confiable y actualizada sobre Ciencia, Tecnología e Innovación que oriente la definición de políticas y la toma de decisiones en materia científica.

La construcción y desarrollo de indicadores de Ciencia y Tecnología es una práctica arraigada en algunas sociedades de Latinoamérica. Los primeros esfuerzos fueron desarrollados en la década de los noventas (UNESCO, 2010). Fue a partir del año 1994 con la creación de la Red Iberoamericana de Indicadores de Ciencia y Tecnología (RICYT) que se inicia a mitigar el vacío de información en materia científica en Latinoamérica. A partir de ese momento la RICYT ha sido la encargada de la ejecución del programa regional de indicadores de Ciencia y Tecnología, quien a través de foros de discusión ha permitido que los países latinoamericanos avancen en various members of the research and innovation system to provide information and feed the system, to generate a software to manage the system, and train the staffs responsible for the systematic collection of information, the analysis, reporting, comparisons with other countries, scientometrics, innovation and public perception of the social impact of science and knowledge.

el diseño de instrumentos para la conceptualización y medición de aspectos específicos que adquiere el proceso de generación y difusión del conocimiento en cada uno de los países de la región (RICYT, sf).

Algunos estudios regionales muestran que en Nicaragua, a pesar del poco fomento a la Ciencia y la Tecnología, la producción científica está siendo reflejada en bases de datos internacionales. Estudios realizados por Torres (2007), muestran la presencia de la producción científica nacional en la web de la ciencia, con una producción de 264 publicaciones de autores afiliados a instituciones nicaragüenses en el decenio 1995-2004. Por otro lado un estudio realizado por Arencibia-Jorge (2011) muestra que según el ranking Iberoamericano de SCIMARGO (Datos de SCOPUS, 1996-2008), en ese periodo se publicaron un total de 529 artículos científicos. De los 529 artículos citados, $48 \%$ corresponde a cinco universidades públicas pertenecientes al CNU (Universidad Nacional Autónoma de Nicaragua (UNAN León), Universidad Nacional Autónoma de Nicaragua (UNAN Managua), Universidad Nacional Agraria (UNA), Universidad Centroamericana (UCA) y Universidad Nacional de Ingeniería (UNI)).

A pesar de éste resultado alentador, se hace necesario disponer de un conjunto de indicadores que brinden información sobre la inversión y gasto en Ciencia y Tecnología, la oferta científica tecnológica nacional, el impacto del gasto público en $\mathrm{C}$ y $\mathrm{T}$ y la forma como este gasto se ha traducido en bienes y servicios para la sociedad nicaragüense.

\section{DESARROLLO}

Que son los indicadores. Los indicadores son parámetros que ponen de relieve una faceta del objeto de la evaluación. Por su naturaleza, la Ciencia necesita de múltiples indicadores para poder patentizar el resultado de la misma. Los indiciadores pueden ser medidas, números, hechos, opiniones o precepciones que señalan situaciones o condiciones específicas.

Los indicadores son instrumentos importantes para evaluar y dar seguimiento al proceso de desarrollo, valiosos para orientar cómo se pueden alcanzar mejores resultados. En el caso de Ciencia, Tecnología e Innovación, los indicadores están vinculados con los procesos de medición de las actividades de generación, uso y difusión del conocimiento científico, el desarrollo tecnológico y la innovación organizacional.

Los indicadores de C, T e I deben ser construidos sobre la base de las visiones y realidades de un determinado país. 
Para qué un sistema de indicadores de ciencia y tecnología e innovación. El propósito fundamental es medir la Ciencia y la Tecnología para valorar el desempeño científico tecnológico de manera integral teniendo en cuenta aspectos económicos, ambientales, sociales, tecnológicos y de investigación científica. Los procesos decisorios que atañen a la Ciencia, Tecnología e Innovación requieres una base amplia de formación cualitativa y cuantitativa acerca de recursos disponibles, resultados alcanzados, tendencias y escenarios futuros. En esencia es contar con mejor información para desarrollar nuevas y firmes políticas de Ciencia, Tecnología e Innovación.

Atendiendo a esta problemática, los indicadores se constituyen en una herramienta fundamental para la evaluación y la planificación de las políticas en este campo. Por tanto, se hace necesario un proceso hacia la creación y posterior consolidación de un espacio permanente de construcción de estadísticas en ciencia y tecnología.

Con la construcción del sistema nacional de indicadores se procura que en Nicaragua se brinden elementos que contribuyan a diseñar mejores políticas de ciencia tecnología e innovación, que coadyuven de manera relevante al desarrollo económico y social de los nicaraguenses.

Antecedentes en Nicaragua. En Nicaragua son escasos los intentos orientados para la definición de indicadores de Ciencia, Tecnología e Innovación. Un primer intento fue el desarrollado por el Banco Interamericano de Desarrollo (BID), en el año 2004, quien a través del proyecto de modernización de la educación terciaria en Nicaragua incluyó en uno de sus componentes la identificación y consenso de nuevas variables e indicadores de investigación y posgrado (CNU, 2004).

En el año 2008, a través del CONICYT se implementó un estudio llamado Directorio Nacional de Ciencia y Tecnología (DNCT), con el propósito de visibilizar la producción científica de las instituciones que hacen investigación en Nicaragua, sin embargo, a pesar de los esfuerzos destinados a esta misión, las instancias decisoras y los actores claves de los sistemas de investigación e innovación jamás tuvieron acceso al resultado final del estudio.

Posteriormente, en el año 2010, las universidades miembros del CNU desarrollaron un ejercicio tendiente a la construcción de indicadores de Ciencias y Tecnología en el ambiente universitario. De este esfuerzo se obtuvo un producto que en la práctica ha tenido muy pocos frutos, más allá de ser uno de los documentos bases para la ejecución del estudio actual.

Proceso de construcción de indicadores de ciencia, tecnología e innovación en Nicaragua. En el presente (2013-2014), CONICYT en coordinación con la comisión de investigación, extensión y estudios de posgrado del CNU están abocados a la construcción del Sistema de Indicadores Nacionales. El paso inicial fue constituir un equipo de trabajo que se hiciera responsable del proyecto, tarea que recayó sobre investigadores del CONICYT e investigadores de la Universidad Nacional Agraria. La responsabilidad de esta misión es potestad del equipo de investigadores, quienes han recibido asesoría y retroalimentación en el proceso de parte de la comisión de investigación, posgrado y extensión del CNU.

El proceso de construcción de dichos indicadores se ha estado realizando a través de la utilización de diversas técnicas y metodologías participativas, las cuales han estado siendo aplicadas por etapas. Las etapas comprenden, elaboración del marco conceptual; formulación de indicadores; elaboración de documento y aproba- ción del documento final. Las propuestas de estas etapas incluyen talleres de socialización para retroalimentar y aprobar el producto.

A la fecha, el trabajo se encuentra en la primera etapa que es la elaboración del marco conceptual. Este inició con un proceso de búsqueda de información, aplicando la técnica de revisión documental en diferentes entidades nacionales e internacionales, realizando visitas in situ o aplicando técnicas de búsqueda en internet. Dicha información facilitará elementos legales y técnicos para describir un contexto legal y un marco conceptual sobre los indicadores de CTI. En la continuación del proceso de esta primera etapa se realizó un taller de socialización con miembros de las universidades y actores claves de los sistemas de investigación e innovación para retroalimentar y aprobar el producto.

La información procedente de la primera fase, muestra la experiencia desarrollada por otras sociedades en la construcción de indicadores de ciencia tecnología e innovación (i.e. indicadores, método de colección de datos, método de análisis, periodicidad de colección de información, resultados y retroalimentación al sistema), la cual ha permitido a los investigadores consensuar la información pertinente para Nicaragua, de acuerdo a nuestro desarrollo y realidad.

El trabajo de esta primera etapa continuó con la elaboración de instrumentos para la recolecta de la información. El equipo de trabajo (investigadores) en coordinación con la Comisión de Investigacion, Extensión y estudios de Posgrado del CNU trabajó en la elaboración de la encuesta que permitieran recoger información veraz de las instituciones académicas, ONGs, instituciones estatales y centros de investigación cuya misión incluyera la formación de talentos humanos y la generación de conocimientos, técnicas y tecnologías. Basado en éste instrumento, se diseñó una plataforma en la aplicación SPSS que pudiera albergar la base de datos que serán generados durante el estudio.

El proceso continuó con la elaboración de un instructivo que recogiera el concepto del sistema de indicadores de CTI y su aplicación como una herramienta de evaluación de los esfuerzos en CTI (i.e. conceptos básicos de indicadores, investigación, innovación, etc., método de colección de datos, método de análisis, periodicidad de colección de información, resultados y retroalimentación al sistema, entre otros).

El proceso de trabajo incluyó la conformación y capacitación de un grupo de investigadores quienes serían los encargado de la recolección sistemática de la información, Los investigadores fueron objeto de entrenamiento en el manejo de los instrumentos y en el manejo del instructivo, y han sido los encargados de visitar cada una de las instituciones seleccionadas en la muestra.

En Nicaragua son pocas las instituciones que poseen en su misión el desarrollo de la ciencia y la tecnología a través del fomento de la investigación como eje fundamental de su quehacer. La valoración inicial sobre la muestra a atender dio pautas para definir la población en su totalidad como objeto de estudio. Al final la población estuvo constituida por 100 instituciones que proveerán información, 57 de ellas son instituciones educación superior. Las restantes son instituciones estatales, organismos no gubernamentales y centros de investigación, algunos de ellos adscritos a universidades.

Es así que basados en la experiencia desarrollada por otras sociedades y con el apoyo de organizaciones que apoyan esta labor en Latinoamérica, se han articulado esfuerzos para avanzar hacia el diseño de instrumentos para la conceptualización y medición de los aspectos específicos que adquiere el proceso de generación y 
difusión del conocimiento en Nicaragua. Para el país será la primera experiencia, la cual lleva ya varios años en otros países latinoamericanos.

La fase final de esta primera etapa deberá incluir la elaboración del marco conceptual que cimente esta primera experiencia inédita para Nicaragua. La definición de la ruta a seguir deberá tener en consideración desarrollar soluciones adecuadas a nuestras propias inquietudes en lo que respecta a la producción, difusión y aplicación del conocimiento, en los contextos de nuestra sociedad y actividad económica.

Etapas concluyentes del proceso. El estudio incluye una segunda etapa que iniciará con la elaboración de instrumentos para la definición de indicadores. Se realizará un proceso de análisis para definir las dimensiones, funciones, factores, entre otros. Luego se procederá a la formulación y definición de los indicadores por categoría. Al finalizar se realizará el taller de socialización para retroalimentar y enriquecer el producto.

Una vez definido y aprobado el marco conceptual, con los indicadores formulados y aprobados, se integraran las partes elaborando un documento preliminar, el cual será revisado y retroalimentado en un taller con actores claves del sistema

La etapa cuatro del estudio lo constituye la aprobación del documento final, la misma inicia con la incorporación de las sugerencias de mejoras brindadas en el taller previo, lo que devendrá en el documento final. El documento será presentado en una sesión participativa, se incorporaran las sugerencias que se hagan durante la sesión de aprobación, para luego preparar el documento final y hacer su entrega oficial.

También son de importancia la implementación de actividades que fomenten en los actores claves del SNIN el interés por la creación del sistema de indicadores de Ciencia, Tecnología e Innovación, esto implica analizar la importancia de la creación del sistema a luz de su aplicabilidad práctica, el intercambio de experiencias sobre la creación del sistema de indicadores con otros países de la región, y en la marcha, compartir el avance del proyecto "Construcción del Sistema de Indicadores de Ciencia, Tecnología e Innovación en Nicaragua"

Es necesaria la creación de compromisos entre los diversos integrantes del sistema de innovación para proporcionar la información y alimentar el sistema, generar un software para administrar el sistema, y capacitar al personal encargado de la recolección sistemática de la información, su análisis, elaboración de reportes, comparaciones con otros países, cienciometria, entre otros. Se necesita información comparable sobre procesos de innovación y bibliometría, así como precepción pública de la ciencia e impacto social del conocimiento.

\section{CONCLUSIONES}

El proceso de construcción de indicadores de Ciencia, Tecnología e Innovación se ha estado realizando a través de la utilización de diversas técnicas y metodologías participativas, las cuales han estado siendo aplicadas por etapas. Las etapas comprenden, elaboración del marco conceptual; formulación de indicadores; elaboración de documento y aprobación del documento final.

La fase final de esta primera etapa deberá incluir la elaboración del marco conceptual que cimente esta primera experiencia inédita para Nicaragua. La definición de la ruta a seguir deberá tener en consideración el desarrollo de soluciones adecuadas a nuestras propias inquietudes en lo que respecta a la producción, difusión y aplicación del conocimiento en los contextos de nuestra sociedad y actividad económica.

La construcción del Sistema Nacional de Indicadores será exitosa en la medida que los actores claves del sistema nacional de investigación e innovación se interesen en la creación del mismo.

En el proceso, es de vital importancia la creación de compromisos entre los diversos integrantes del sistema de investigación e innovación para proporcionar la información y alimentar el sistema.

Se hace imprescindible generar un software para administrar el sistema de indicadores, y capacitar al personal encargado de la recolección sistemática de la información. Es necesaria la creación de compromisos entre los diversos integrantes del sistema de innovación para proporcionar la información, alimentar el sistema y capacitar al personal encargado de la recolección sistemática de la información, análisis, elaboración de reportes, comparaciones con otros países, cienciometria, entre otros. Se necesita información comparable sobre procesos de innovación y bibliometría, así como precepción pública de la ciencia e impacto social del conocimiento.

Se necesita implementar actividades que fomenten en los actores clave del SNIN el interés por la creación del sistema de indicadores de Ciencia, Tecnología e Innovación. Esto implica analizar la importancia de la creación del sistema de indicadores a luz de su aplicabilidad práctica, el intercambio de experiencias con profesionales de otros países que hayan desarrollado el proceso, y en la marcha, compartir el avance del proyecto en Nicaragua.

\section{AGRADECIMIENTO}

Los autores agradecen el acompañamiento brindado en el trascurso de esta investigación de los licenciados Moisés Gómez, Alberto García, Oscar Moreira y Saray Gaitán del Consejo Nicaragüenses de Ciencia y Tecnología (CONICYT), al arquitecto Benjamin Rosales (Universidad Nacional de Ingeniería), Lic. Mario Arguello (Universidad politécnica de Nicaragua), Lic. Santos Solórzano (Consejo Nacional de Universidades), José Antonio Chavarría (UNAN-León) y Lic. Anielka Pérez (Universidad Centroamericana). 


\section{REFERENCIAS BIBLIOGRÁFICAS}

Arencibia-Jorge, R; Araujo-Ruíz, JA; Hung Llamos, BR Alemán-Zeledón, FS; Chinchilla-Rodríguez, Z; Hernández-Cañadas, P; Hoz de Villa Barbery, R; Sheehan, T; Díaz-Mayans, C; Belcher, M; de Moya-Anegón. F. (2011). Bibliometric study of Latin American countries supported by INASP 1996-2008. INASP. Oxford. UK. 81 p

CNU (Consejo Nacional de Universidades). 2004. Variables e Indicadores Estadísticos de Investigación y Posgrado. Resultado Final de la Ejecución del Proyecto BID. 18 p.

CONICYT (Consejo Nicaragüense de Ciencia y Tecnología). 2010. Plan nacional de ciencia tecnología e innovación (2010-2013). 67 p.

CONICYT (Consejo Nicaragüense de Ciencia y Tecnología). 2008. Directorio nacional de investigación y desarrollo. Resumen ejecu tivo y análisis de resultados. $13 \mathrm{p}$.

RICYT (Red de Indicadores de Ciencia y Tecnología Iberoamericana e Interamericana). Que es la RICYT. http://www.ricyt.org/quees-la-ricyt.

Torres, GE. Situación de la investigación universitaria en Nicaragua. 2007. En: Situación de la investigación universitaria centroame ricana. p 101-124. CSUCA. IESALC. 194 p.

UNESCO (Organización de las Naciones Unidas para la Educación la Ciencia y la Cultura). 2010. Sistemas nacionales de ciencia tecnología e innovación en América Latina y El Caribe. Estudios y documentos de política científica en ALC, Vol. 1. Oficina re gional de ciencia para América Latina y El Caribe. Edificio MERCOSUR. 324 p. 